\title{
Ethnoveterinary knowledge in pastoral Karamoja, Uganda
}

\author{
Jeanne Gradé
}

Supervisor: Patrick Van Damme

Laboratory of Tropical and Subtropical Agriculture and Ethnobotany, Ghent University

Public defence: 12 December 2008

The present study presents the cataloguing and documenting of indigenous veterinary knowledge of Karamojong pastoralists as a component to an ongoing nongovernmental organisation-managed community animal health and development program. The region of Karamoja, $28,000 \mathrm{~km} 2$, is located in northeastern Uganda, in the Great Lakes Region of East Africa.

The following problem statements justify this research. Karamoja has a fragile indigenous knowledge data base, prone to fragmentation. It is primarily oral history with very little written data. The growing number of locally endangered medicinal plants presents another and related problem. The area has high (small arms) insecurity and deforestation indices. Since the Karamojong people are semi-nomadic pastoralists with limited agriculture experience, they have little familiarity with agroforestry, plant propagation, resource conservation and protection. Karamojong people have a marginalised lifestyle. Their region has poor infrastructure with high mortality and morbidity rates. The area is prone to recurrent drought and famine. Government relationships are poor, adding to the external/ environmental pressure that Karamojong knowledge faces. Finally, Karamojong culture and lifestyle is focused on cattle, yet they have the poorest veterinary services available in Uganda. Karamojong indigenous knowledge (IK) has proven remarkably resilient in the face of inhospitable environments, less so regarding the social pressures mentioned above. The ecological environment has become harsher and grazing areas more limited during the lifetime of the elders. As a result of these environmental changes, we see increasing reliance on external support and foreign elements entering the system, thereby diluting their indigenous coping mechanisms.

Cataloguing the indigenous veterinary treatments began ten years ago, with the overall objective of integrating the most confidently used treatments into regional development livestock trainings. The next phase of research and development (R\&D) entailed selecting a few of these plant treatments to promote in agroforestry and in scientific validation field trials. During this field trial phase, goats were observed to self-medicate. As very little is known about animal self-medication, we set up another study to better understand livestock zoopharmacognosy. Continued research activities revealed the depth and breadth of Karamojong IK. Meanwhile, external shareholders got another glimpse into IK's potential for community and community animal health worker (CAHW) local capacity building. The research blended endogenous with exogenous ideas and R\&D networks. Endogenous approaches mingled both science and tradition, thereby adding 
value to both. This phase included ways to 're-discover', re-invent and make Karamoja's ethnoveterinary knowledge (EVK) systematically available for community use. The final phase investigated how well this blended EVK diffused into the community.

The pastoralists of south and central Karamoja have a lot of indigenous knowledge and a variety of plants to choose from to satisfy their subsistence requirements and to cater to their livestock healthcare needs. This study recorded EVK information on 209 plant species, distributed over II 6 genera and 54 families. There were 130 separate EVK uses listed. The most common indication was against anaplasmosis, for which 29 species were reported. Many of the plants in this inventory were never documented for animals before at all and/or for the specific uses recorded here (Chapter III).

Due to its local importance, a field trial case study is illustrated, testing a traditional dewormer medicine, Albizia anthelmintica, under field conditions. The EVK treatment proved to be effective at levels that are consistent with the veterinary pharmaceutical standards for a novel treatment. Therefore, Karamojong EVK holds potential for developing sustainable local resource-based and integrated livestock management plans not only in the study area, but also in other developing countries (Chapters IV, V).

The thesis assessed not only the pastoralists' wisdom, but also the livestock's 'intuition', where the hypothesis that animals self-medicate was investigated, by using a combination of veterinary, ethnobotanical and indigenous research techniques. We showed that livestock evidently self-medicate. There is reason to suggest, that in Karamoja, some ethnomedicine knowledge has originated from careful animal observation. To our knowledge, this was the first time the techniques we used were combined in zoopharmacognosy, and one of the few studies ever to investigate livestock self-medication (Chapters IV, VI).

Results of ethnographic action research show that with growth of a viable EVK network in Karamoja, local interest for nature conservation has increased. Encouraging EVK and increasing medicinal plant availability benefits not just livestock, but also the people who depend on them. The sharing has stimulated dialogue between antagonistic groups, within families, clans and tribes and even across borders. Strengthening local institutions that address EVK and natural resource management creates a context for peace as a by-product (Chapter VIII).

Ethnoveterinary knowledge is more commonly known and used where healers have actively shared it with one another. The latter would indicate that registered healers have shared their EVK effectively and have encouraged its diffusion in the areas studied. These results support the working hypothesis that knowledge will not disappear if it is used and communicated (orally, practically and written) through all available networks - indigenous, endogenous and exogenous. This may enable a smoother transition into the next cultural identity era in which Karamoja reaches a sustainable independent way of living in relation to the modern context (Chapter VIII).

This written body of research presents Karamoja's ethnoveterinary knowledge and also validates the pastoralists' ethnobotany knowledge through systematic scientific research. It has both substantiated and strengthened this knowledge and has opened more 
avenues for further validation, a sound foundation for development initiatives.

Given livestock's central role in Karamoja, we recommend that community development programs have an initial EVK survey as an integral part of their core methodology, dynamics and approach. The work in this thesis contributes significantly to the understanding that EVK is at the heart of Karamojong culture. Efforts to preserve, promote and protect it will benefit the entire culture.

This ethnoveterinary study, in a previously unstudied area and with a poorly documented population still living in a traditional way, has helped add to the growing body of knowledge about useful plants in Uganda. The present study safeguards the EVK availability for future generations of the traditional communities concerned, for the entire socio-cultural Karamoja cluster (southeast Sudan, northwest Kenya, and southwest Ethiopia where other closely-related ethnic groups still practice a transhumant lifestyle), as well as for the R\&D community at large. 